and pain in both temporal regions rapidly followed by cedematous swellings of the size of an orange; the next day there was no trace of these swellings beyond soreness at their site.

From this date (the 3rd) there followed a rich abundance and variety of symptoms. From hour to hour, not merely from day to day, the clinical picture would change in a manner which defies description but which soon reduced the boy's attendants to a state of stolid but sympathetic expectancy and the patient himself to complete indifference. He seldom spoke throughout his long illness and even his monosyllabic utterances were accompanied by tears. In the course of 24 hours ugly swellings would appear and disappear, a dozen joints would be racked with pain in rapid succession, and the surface of the body would exhibit a whole atlas of skin diseases. Simple erythema and purpura, urticaria, and erythema exudativum in all its varieties, cropped out and disappeared for week after week in disheartening fashion; blushes and spots, stony nodes and nodules, and enormous papules presenting themselves and vanishing one after another or in groups, but always irregularly in order and seat.

On the 4th there was occasional colic; on the 5th the most prominent features were cedema of the right upper eyelid of the size of a hen's egg, and in the evening return of the colic and vomiting, and severe pain in the elbows, wrists, and ankles, while both cheeks swelled suddenly, and erythema nodosum and purpura contended for supremacy in the limbs. Purpura was triumphant on the morning of the 6th and the cdema was transferred from the cheeks to the forehead as though a variety of grotesque distortions of the features were the object ; in the afternoon the buttocks were covered thickly with erythema papulatum. But the colic remained the most anxious symptom up to March 8th when it subsided in favour of pain all over the back, and this gave place in turn to pain, œdema, and tenderness of the left side of the trunk, the left thigh, knee and hand, and both legs, causing inability to move. The patient was able to move comfortably once more by 9.30 P. M. On the 9 th and he had no swelling save over one knee and one shoulder, and he was able to sleep well. At 1 A.M. on the 10th he awoke with great abdominal pain and vomiting resulting in an alarming collapse which caused several anxious hour and required active measures. By midday the colic had gone, but a copious purpuric rash had appeared on the face and elbows. From the 12th to the 15th the colic decreased the flying pains and swellings continued until the 13th, and the only fresh rash was purpuric on both cheeks, the left knee, arm, and hand. The 14th was the first day that could be called satisfactory; the patient felt better, there were no pains or swellings, and the skin affection was limited to small crops of purpura. At 8 A.M. on the 15th he was desquamating "all over," but by 1 P.M. his arms were thickly covered with purpura from shoulder to wrist. From this date until the 19th no new features arose, although the old symptoms of pain, swelling, and rash rang the changes on the joints and the different areas of the body, but with less vigour. On the 19th for a brief period there were no pain, no colic, no new rash, a clean tongue, and a normal temperature in the patient, and in his attendants some hope of permanent improvement, when suddenly his flushed face drew attention to the pulse and temperature. Without apparent cause the pulse had risen to 104 and the temperature to $99 \cdot 4^{\circ} \mathrm{F}$. ; there were no abnormal cardiac signs. Up to this time the pulse had been the one cheerful feature, steady at 80 and rarely up to 90 , and of good character, while the temperature had never exceeded $99^{\circ}$. In the next few days the temperature rose slowly to $100 \cdot 4^{\circ}$ of an evening and $100^{\circ}$ in the morning, while the pulse-rate varied day and night between 120 and 130 . On the 22 nd the old symptoms took a new lease, colic and purpura, urticaria, cedema, and erythema, sudden and vanishing pains and swellings, once more perplexing us. On the 27th it was noted that the pains had left the region of the great joints and were affecting only the hands and feet, the axilla and popliteal spaces, and the muscles of all the limbs. On the 28th there was actually no pain, swelling, or rash, only vigorous desquamation once more; but the increased pulserate and temperature warned us not to draw encouragement again from the suspension of the original symptoms. The patient had a restless night; at $11 \mathrm{~A} . \mathrm{M}$. on the 29 th the pulse was 136 and the temperature was $99.4^{\circ}$ and for the first time a loud systolic murmur was detected in the mitral area without any increase of cardiac dulness. The cardiac condition now became the chief anxiety; there was, from this time, no return of the original symptoms except that the skin eruptions, especially the purpura, recurred every night for several weeks. It was interesting to observe that an erythematous blush could be produced at any time on any portion of the chest and abdomen simply by letting the patient know that one's attention was directed to that portion. The pulse-rate fell slowly to about 106 by April 26th, on which day the temperature reached normal. The cardiac dulness remained unaffected. By May 4th the pulse was under 100 and on the 27th he was able to watch the school sports from his window, although he paid for the pleasure by a large crop of purpura on his legs and a slightly raised temperature-90 days after the onset of his illness; but small crops of purpura were not uncommon five months after the date of onset of his illness. The convalescence was marked by a gradual lessening in intensity of the cardiac murmur, encouraging a hope that it would ultimately disappear; but once more a new development retarded recovery. Scarcely had he begun to use his legs when distinct, but happily not severe, chorea set in, the head, neck, and upper extremities, as well as the face, being affected mostly. This condition persisted for several months but did not become serious; indeed, the boy was able to make a voyage to South Africa during the winter. I have been unable to obtain a report as to his present condition but he is said to be well although " nervous" as before.

During the illness examination of the blood gave no information. Frequent examinations of the urine detected no renal mischief. During the first three weeks of the illness the total daily flow of urine was diminished more than there appeared reason for ; it averaged, during that period, 18 ounces and ranged from nil to 29 ounces per diem. No benefit was obtained from the use of salicylates or any of the numerous drugs selected out of recommended remedies. Henoch's recommendation of icebags to the abdomen for the colic was of no avail. Careful nursing and feeding, together: with palliative treatment of symptoms as they arose-a proceeding which often recalled the carpenter's estimate of the prospects of the seven maids with seven mops-was the method of treatment employed in this case.

No doubt the case presented a curious jumbling-up of the symptoms of "Henoch's purpura" and "purpura rheumatica" with additions. The transient earache on Feb. 23rd has. been suggested as a possible source of septic infection. The suggestion is incapable of proof or disproof. Whatever the poison it appeared to act upon the nervous system.

The case differed from those described by Henoch as

follows : (1) In its more continuous nature ; there were no "intervals of several days or weeks or even of a year" (Henoch); (2) in the prominence of skin eruptions other than purpura, and of what can only be called "flying oedema" (3) in the cardiac complication ${ }^{2}$; (4) in the chorea; and (5) in the diminished daily flow of urine.

I had the advantage of meeting Dr. H. P. Hawkins in consultation over this case. Florence.

\section{A CASE OF APPENDICITIS WITH GENERAL PERITONITIS.}

BY W. GREENWOOD SUTCLIFFE, F.R.C.S. ENG., HONORARY SURGEON TO MARGATE COTTAGE HOSPITAL AND THE ROYAL SEA-BathiNg hospitaI, MaRgate.

RECOVERY of cases of this gravity being sufficiently rare as compared with the small mortality attending opera. tions where the damaged part is only slightly shut off, the details of this case may be worth putting on record.

The case appears to have begun on Oct. 24th, 1902, the patient, a girl, aged 20 years, being seized on that night with violent abdominal pain which was treated in the usual domestic manner without medical advice by the application of hot fomentations, with the result that the pain subsided next day, and a similar attack having occurred 12 months before which had cleared up satisfactorily it was thought that the trouble was practically at an end. The patient was able partially to go on with her housework she, however, remained constipated and slight pains in the

Compare a case of purpura described by Dr. C. W. Buckley The Lancet, July 20th, 1901, p. 132 
right ileo-cral region persisted; she therefore took a large dose of castor-oil on the afternoon of the $28 \mathrm{th}$ with the result that the bowels acted freely, but the pains returned with greatly increased severity and there were several attacks of romiting. The girl, of course, was sent to bed again and Dr. A. Warwick Brown was called in late at night. He at once recognised the case as one of severe appendicitis and arranged for her removal to the Margate Cottage Hospital next day. He saw her next morning and finding that the area of tenderness had greatly increased and that the girl was distinctly worse caused her to be taken to the hospital with great care on an ambulance. She was seen by me an hour after admission. There were then well-marked signs of general peritonitis, the pulse was over 180 , the temperature was $101^{\circ} \mathrm{F}$., the abdomen was rigid all over, and no movements were visible with respiration, which were shallow and over 40 to the minute. There were occasional ineffectual attempts at vomiting. The girl was too bad to elicit where the area of greatest tenderness was to be found, and in view of her collapsed condition we considered the outlook to be distinctly unfavourable.

An operation was decided on and carried out as rapidly as possible, Dr. Brown administering chloroform and Dr. R. Thomson assisting me. The usual oblique incision over the region of the appendix was made and on opening the peritoneum a quantity of thin pus escaped; this was not offensive and there were no adhesions. The cæcum and appendix which was behind it and directed inwards were exposed, and round the base of the creum there was some thick white lymph, evidently of some days' standing, and a piece of swollen, inflamed, and cedematous omentum of the size of a visiting card was adherent to the appendix, which was itself swollen, intensely injected, and perforated at its tip. It was thus quite clear what had happened : the attack had started five days before and the inflamed area was being shut off in the usual manner, but the morbid products had been let into the general peritoneal cavity as the result of the purgative and the consequent violent peristalsis. The appendix and damaged omentum were rapidly removed and an incision two inches long was made in the middle line; through this a large glass irrigator nozzle was passed and a quantity of thin odourless pus welled up from the bottom of Douglas's pouch. A stream of hot saline solution was then directed through the tube and made to flow out through the lateral opening until the solution returned quite clear, when a large rubber drainage-tube was passed through the median incision reaching to the bottom of the pelvis and a similar tube was passed upwards through the lateral incision by the side of the crcum. Both incisions were then lightly plugged with sterile gauze and left wide open, no sutures being inserted. The usual sterile dressings were applied and the patient was got back to bed as rapidly as possible. She stood the operation well, it having taken 40 minutes. On the next morning there was a marked improvement in the condition of the patient, the temperature had fallen to $99^{\circ}$ and the pulse to 110 and there had been no more attempts at vomiting. There was a fair amount of discharge from each tube and during the night of the operation and the following day the dressings were frequently changed; the gauze plugging was changed at the end of 24 hours. On the 31st, two days after the operation, the improvement continued; the temperature was $99^{\circ}$ and the pulse was 100 . The rigidity had entirely, and the tenderness nearly, disappeared; the abdominal walls moved freely with respiration; and the discharge from the tubes having practically ceased they were removed 48 hours from the time of operation. The wounds were after this lightly plugged twice daily until no longer necessary, and from then to the time she left the hospital, four weeks later, with both wounds practically healed, her return to convalescence was uneventful and uninterrupted.

I regret that a bacteriological examination of the pus was not made, but both symptoms and appearances pointed to one of the worst forms of septic peritonitis and I hardly dared to hope for a recovery. Possibly it was due to the fact that the intestines, with the exception of the washingout, were disturbed as little as possible. There is no doubt that their removal for cleaning and consequent handling must add greatly to the shock and so places the patient's tissues at a disadvantage in dealing with the organisms that are certain to be left behind in spite of the most careful "toilet." Leaving the wounds open in these acute cases not only facilitates drainage but has another great advantage -the patient is sooner off the table and the period of convalescence, if the wounds are carefully dressed, is very slightly prolonged. The other factor in the girl's recovery was the promptitude of my friend Dr. Brown in advising surgical interference, as even with all the publicity afforded to the disease in the last few years the aid of active surgery is too often invoked too late. Margate.

\section{HÆMORRHAGE IN PREGNANCY : A CASE OF CENTRAL PLACENTA PRAVIA.}

By H. T. M. WhiTliNG, M.B., B.S. Durh., M.R.C.S. ENG., L.R.C.P. LOND.

ON Nov. 15th, 1902, the nurse in attendance on a married woman sent for me at 7 A. M., stating that labour pains had commenced about three hours previously and that they had been attended before and during her (the nurse's) presence with alarming "flooding." The patient, aged 40 years, was a multipara, with five children, whom I had twice previously attended at confinements (once for twins) and whom I knew to be generally quick in labour and the possessor of a large pelvis and an unrepaired ruptured perineum dating from her first confinement, which gave plenty of vaginal room as it extended rather far back towards the rectum. When I arrived there were numerous signs-such as clots, \&c. - on the bed of a severe hæmorrhage and a utensil was nearly half filled with fresh blood which had just been passed while micturating.

On examination per vaginam the os was found to be slightly larger than a five-shilling piece and was apparently soft and easily dilatable. Blood was flowing freely and my finger perceived the almost unmistakeable "boggy" soft sensation caused by a presenting placenta. The membranes were intact and bimanually I made out the back of the foetus to the right and the limbs to the left of the uterus; the vertex was right occipito-anterior. Having swept two joints of my examining finger round inside the os uteri and thereby separated the placenta as far as possible, I found that without passing $m y$ whole hand into the vagina and through the os I should not be able to reach the edge of the placenta to rupture the membranes, so I determined to tear strajght through the placenta itself, seize a leg or foot, and to turn quickly, or, if the head was found low down, as I thought to be the case, to apply forceps and to deliver rapidly; for celerity in the latter procedure I knew that the pelvis and vagina afforded plenty of space and no obstruction and forceps had been used by me before.

Withdrawing my hand I prepared a douche of izal and water at a temperature of $115^{\circ} \mathbf{F}$, injected five grains of "ergotin aseptic" hypodermically into the gluteal muscles, and got $\mathrm{my}$ forceps ready. Pains were now fairly severe and bleeding was free, but the general condition was still not very bad and as my whole attention was necessary for other matters chloroform was not used, the patient remaining quiet and obedient. Re-inserting my hand into the vagina I placed the first and second fingers on the presenting surface of the placenta and, fixing the body of the foetus externally with my left hand, with very slight resistance I tore through the placenta and, passing all my fingers through and widely enlarging the tear to give room, I felt the head presenting and strongly forced down by uterine action a tergo. Changing hands, and dismissing all ideas of attempting podalic version, I rapidly applied my forceps and delivered the foetus exactly three and a half minutes from taking up the first blade, bleeding ceasing for the first time when traction commenced. Passing up my hand without any delay as the child's legs escaped I removed the placenta, firmly compressing the uterus with my left hand. The placenta came away in the hollow formed by my fingers and the membranes dragged after. Then two hot intra-uterine douches of solution of izal were given and with the uterus contracting well no further hæmorrhage occurred.

The child was, as was fully expected, dead and of apparently quite full weight and size. The placenta was very large, thic $k$, and soft, with the aperture made by me for the passage of the hand and foetus almost exactly in the centre, judging by the fairly equal thickness of its substance forming now the circumference of the aperture. Its 